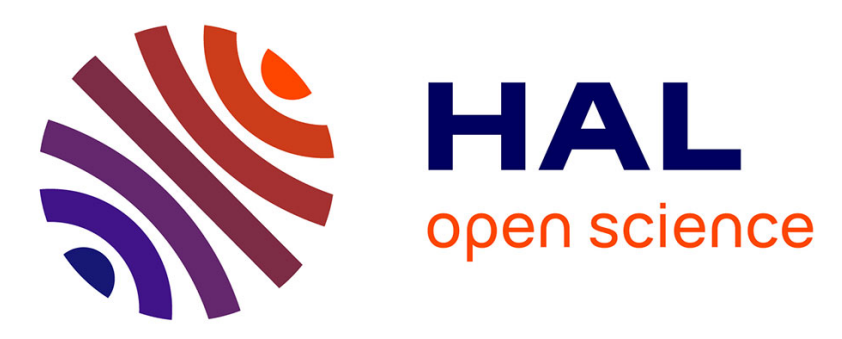

\title{
Size-Specific Spin Configurations in Single Iron Nanomagnet: From Flower to Exotic Vortices
}

Christophe Gatel, Francisco Javier Bonilla, Anca Meffre, Bénédicte

Warot-Fonrose, Etienne Snoeck, Bruno Chaudret, Lise-Marie Lacroix, Thomas Blon

\section{To cite this version:}

Christophe Gatel, Francisco Javier Bonilla, Anca Meffre, Bénédicte Warot-Fonrose, Etienne Snoeck, et al.. Size-Specific Spin Configurations in Single Iron Nanomagnet: From Flower to Exotic Vortices. Nano Letters, 2015, 15 (10), pp.6952 - 6957. 10.1021/acs.nanolett.5b02892 . hal-01707143

\section{HAL Id: hal-01707143 \\ https://hal.science/hal-01707143}

Submitted on 13 Feb 2018

HAL is a multi-disciplinary open access archive for the deposit and dissemination of scientific research documents, whether they are published or not. The documents may come from teaching and research institutions in France or abroad, or from public or private research centers.
L'archive ouverte pluridisciplinaire $\mathbf{H A L}$, est destinée au dépôt et à la diffusion de documents scientifiques de niveau recherche, publiés ou non, émanant des établissements d'enseignement et de recherche français ou étrangers, des laboratoires publics ou privés. 


\title{
Size specific spin configurations in single iron
}

\section{nanomagnet: from flower to exotic vortices}

C. Gatel ${ }^{1, *}$, F.B. Bonilla ${ }^{2}$, A. Meffre ${ }^{2}$, E. Snoeck ${ }^{1}$, B. Warot-Fonrose ${ }^{1}$, B. Chaudret ${ }^{2}$, L.-M.

\author{
Lacroix $^{2}$, T. Blon ${ }^{2, *}$
}

${ }^{1}$ Centre d'Elaboration de Matériaux et d'Etudes Structurales, CEMES-CNRS, 29 rue Jeanne Marvig, B.P. 94347, 31055 Toulouse, France

${ }^{2}$ Laboratoire de Physique et Chimie des Nano-Objets, LPCNO, UMR5215 INSA-UPS-CNRS, Université de Toulouse; Institut National des Sciences Appliquées, 135 avenue de Rangueil, 31077 Toulouse, France

TOC graphic

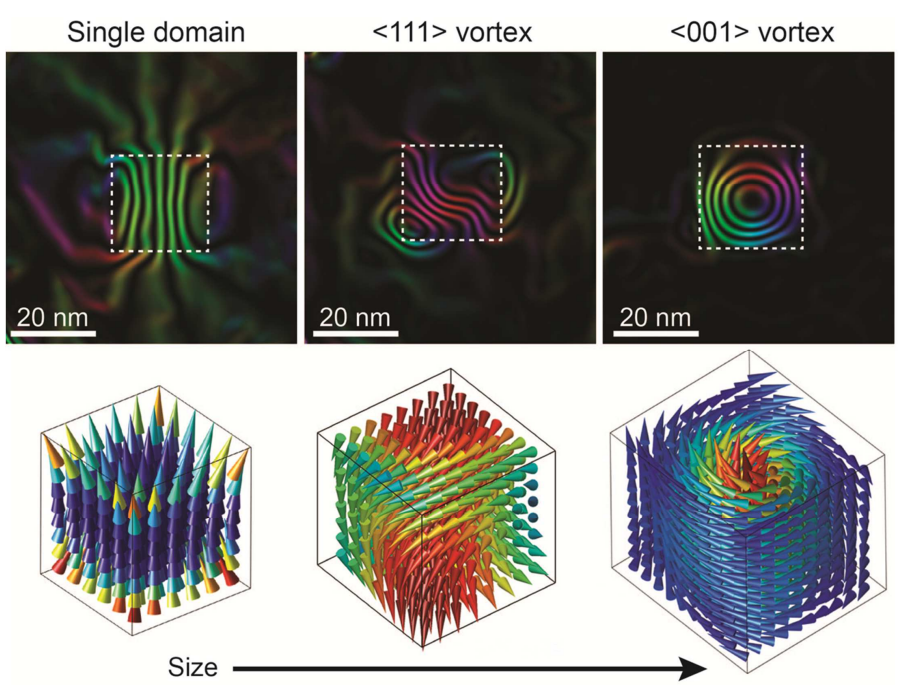




\begin{abstract}
The different spin configurations in the vicinity of the single-domain/vortex transition are reported in isolated magnetic nanoparticles. By combining chemical synthesis, electron holography in a dedicated transmission electron microscope and micromagnetic simulations, we establish the "magnetic configuration vs size" phase diagram of Fe single-crystalline nanocubes. Room temperature high resolution magnetic maps reveal the transition between single-domain and vortex states for $\mathrm{Fe}$ nanocubes from 25 to $27 \mathrm{~nm}$ respectively. An intermediate spin configuration consisting of an $<111>$ vortex is for the first time evidenced.
\end{abstract}

Keywords: magnetic configuration, electron holography, vortex state, single domain state, nanocube.

The magnetic configuration in a magnet of a given volume results from the minimization of the total energy, involving magneto-crystalline anisotropy, exchange and magneto-static energies. As a result, three typical magnetic configurations can be encountered in a magnetic material at the remnant state: (i) a uniform arrangement of magnetic moments, i.e. a singledomain (SD) configuration (Fig. 1(a)); (ii) a vortex (V) state in which external spins rotate to achieve a flux closure, while in the vortex core, spins tilt out-of-plane (Fig. 1(b)); and (iii) a multidomain state dealing with adjacent domains in large volumes. Well characterized in bulk materials, the multidomain configuration is now pursued in magnetic nanowires to benefit from domain wall motion for data storage in the so-called magnetic racetrack memories. ${ }^{1,2}$ While single domain configuration is often optimized for applications requiring hard magnetic behavior, such as hard disk drives ${ }^{3,4}$ or permanent magnets, ${ }^{5,6}$ vortex state is sought in biological 
applications such as hyperthermia or drug delivery ${ }^{7}$ to minimize the stray field around the particle and thus prevent magnetic aggregation, ${ }^{8}$ or in spin-torque vortex-oscillator devices for microwave signal-processing applications. ${ }^{9}$

In these contexts, one needs to evidence at room temperature the single-domain limit separating SD and V states. The determination of this single-domain limit in a single nanoparticle has been largely investigated numerically and is predicted to be in the order of several exchange lengths, i.e. from 15 to $25 \mathrm{~nm}$ depending of the material. ${ }^{10-15}$ Though spin configurations were experimentally determined in different nanomagnets such as nanowires, ${ }^{2,16,17}$ nanocubes, ${ }^{18,19}$ nanospheres,${ }^{20-22}$ faceted nanoparticles, ${ }^{23}$ or nanorings, ${ }^{24-25}$ no result has been reported on the experimental determination of the critical size, even for nano-objects with the simplest geometries. This is mainly due to the limited sensitivity and resolution of existing experimental techniques, which do not allow studying isolated nanomagnets of size close to the expected transition. For instance, magnetic force microscopy ${ }^{26,27}$ and photoemission electron microscopy, ${ }^{28}$ which are classically used for magnetic thin films characterizations, display spatial resolution of few tens of nanometers, far above the single domain limit. Low-temperature spinpolarized scanning tunneling microscopy with spatial resolutions of few angstroms has been used to characterize vortex configurations in elongated Fe islands of several hundreds of $\mathrm{nm} .{ }^{29}$ Vortex states have also been studied in Fe nanocubes of several tens of $\mathrm{nm}$ by off-axis electron holography $(\mathrm{EH})$ in a transmission electron microscope (TEM), with few nanometers of resolution. $^{18,19}$

EH is a powerful TEM interferometric method which consists on superimposing a highly coherent electron beam that has interacted with an object and the surrounding electromagnetic fields, called "object wave", with a part of the same electron beam that has not interacted with 
any field, the so-called "reference wave". The analysis of the resulting interference pattern (i.e. the electron hologram) allows the extraction of the phase shift between the reference beam and the one that has interacted with the magnetic induction through the Aharonov-Bohm $(\mathrm{A}-\mathrm{B})$ effect. The analysis of the magnetic phase image provides a quantitative mapping of the magnetic field with a spatial resolution down to one nanometer. It is important to note that the measured magnetic phase shift corresponds to a projection and an integration of all in-plane magnetic field contributions along the electron path. Numerical simulation are generally necessary to take into account the two dimensional projection of the three dimensional field. (see Supplementary information). Experiments have been performed on an EH dedicated TEM fitted with a cold field emission gun to achieve a high phase shift sensitivity, and a special corrected Lorentz mode allowing a magnetic field-free sample environment and a spatial resolution down to $0.5 \mathrm{~nm}$ (see Supporting Information).

Studying the SD/V transition required a peculiar sample preparation. Isolated nano-objects are mandatory to prevent any influence of dipolar interactions on the magnetic configuration of the object under study. Moreover, defect-free nanomagnets with controlled and reproducible magnetic properties should be sought to allow accurate modeling and comparison with experimental investigations. Finally, simple shape, such as cubes, should be favored to control the nano-object orientation once deposited at the surface. Indeed, in contrast to spheres,${ }^{20}$ cubes can lay on a surface along one of their face, orienting their crystallographic structure for TEM observations and their magnetization for magnetic imaging.

Single crystalline $\mathrm{Fe}$ nanocubes (NCs) were synthesized in solution following an organometallic chemistry approach (details in the Supporting Information). By varying the experimental conditions, cube mean sizes were tuned between 7 and $90 \mathrm{~nm} \cdot{ }^{30}$ Magnetometry 
performed on powders of NCs showed that, whatever the size, the Fe NCs exhibit the bulk saturation magnetization $\left(\mathrm{M}_{\mathrm{S}}\right) \cdot{ }^{30} \mathrm{Fe}$ nanocubes of $27 \pm 4 \mathrm{~nm}$ were deposited on carbon grid by drop casting leading to assemblies of few NCs down to isolated NCs as shown in Figure 1(c-d). High resolution TEM experiments confirm that these nanocubes exhibit a single-crystal bodycentered cubic $(b c c)$ structure with $\{100\}$ facets and <100> edges, the latter being their magnetocrystalline easy axis (Fig. 1(d), Supporting Fig. S1). A $\sim 1 \mathrm{~nm}$ layer of iron oxide shell (most likely $\mathrm{Fe}_{3} \mathrm{O}_{4}$ ), results from air exposition during sample transfer. The determination of the specific shape and magnetic volume being of key importance for accurate measurements of the SD/V limit, the thickness (noted $c$ ) of the NCs should be investigated. TEM images give only access to the lateral lengths $(a, b)$ of the NCs, with about $1 \mathrm{~nm}$ accuracy in EH configuration. Therefore, we evaluated the squareness distribution, defined as $a / b$ ratio, and assumed a similar thickness to length $c / a$ ratio to finally estimate the NC thickness. Squareness distribution of $17 \%$ (Supporting Fig. S1) led to a thickness estimation $c=(1 \pm 0.17) \times a$. For comparison with experiments, micromagnetic simulations were performed using the 3D OOMMF package, ${ }^{31}$ considering single crystalline Fe NCs with $\{001\}$ facets using Fe bulk values for exchange, saturation magnetization and magnetocrystalline anisotropy (details in the Supporting Information). A $1 \mathrm{~nm}$ thick oxide shell was introduced using $\mathrm{Fe}_{3} \mathrm{O}_{4}$ bulk parameters. 


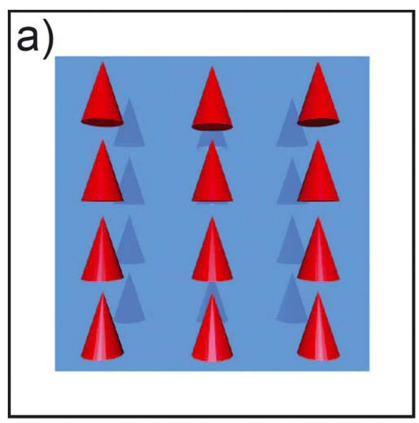

c)

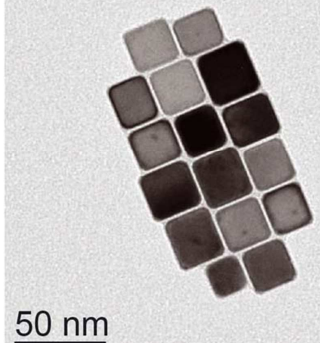

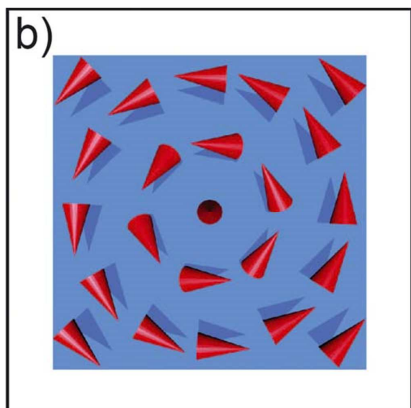

d)

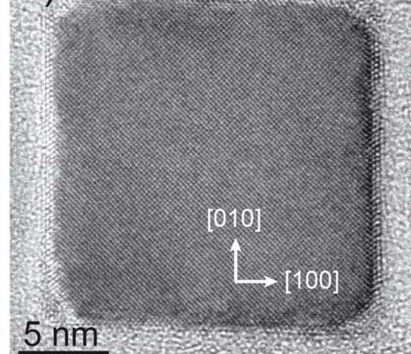

Figure 1. Schematic 2D view of (a) a single-domain and (b) a vortex spin arrangement in a square magnetic element. (c) Transmission electron microscopy (TEM) micrograph of Fe nanocubes. (d) High resolution TEM micrograph of a single Fe nanocube.

Several iron nanocubes have been studied by EH to observe the most probable magnetic configurations. As the NCs have never been exposed to any magnetic field, the measured configurations correspond to virgin remnant states. Three different configurations will be detailed hereafter. Figure 2(a) and Figure S2 of the Supporting Information display the holograms obtained for two different Fe cubes. Figure 2(b) shows the magnetic phase shift map obtained on a $27 \times 26 \times(27 \pm 5) \mathrm{nm}^{3}$ nanocube after separation of the electrostatic contribution (see Supporting Information). For such NC, the magnetic induction curls within the nanocube, evidencing a vortex configuration whose core axis is along the [001] direction parallel to the electron beam, referred to from now on as $<001>$ vortex $(\mathrm{V}<001>)$. To corroborate these 
observations, micromagnetic simulations were carried out on a corresponding $\mathrm{NC}$ of $29.5^{3} \mathrm{~nm}^{3}$, i.e. a $27.5^{3} \mathrm{~nm}^{3} \mathrm{Fe} \mathrm{NC}$ surrounded with a $1 \mathrm{~nm} \mathrm{Fe}_{3} \mathrm{O}_{4}$ shell. Starting from a random spin configuration, the simulation leads to a stable $\mathrm{V}<001>$ state as shown in Figure 2(e). From the calculated magnetization and dipolar field, the total induction $\boldsymbol{B}$ in and outside the NC is obtained and further integrated along the electron beam direction, i.e. the $z$ direction. The simulated magnetic phase shift image of Fig. 2(d) is then calculated thanks to the AharonovBohm relation using the in-plane components of $\boldsymbol{B}$ (details in Supporting Information). A very good agreement between simulated and experimental phase shift maps is obtained, as evidenced by the quantitative matching of the extracted profiles (Fig. 2(f)). 

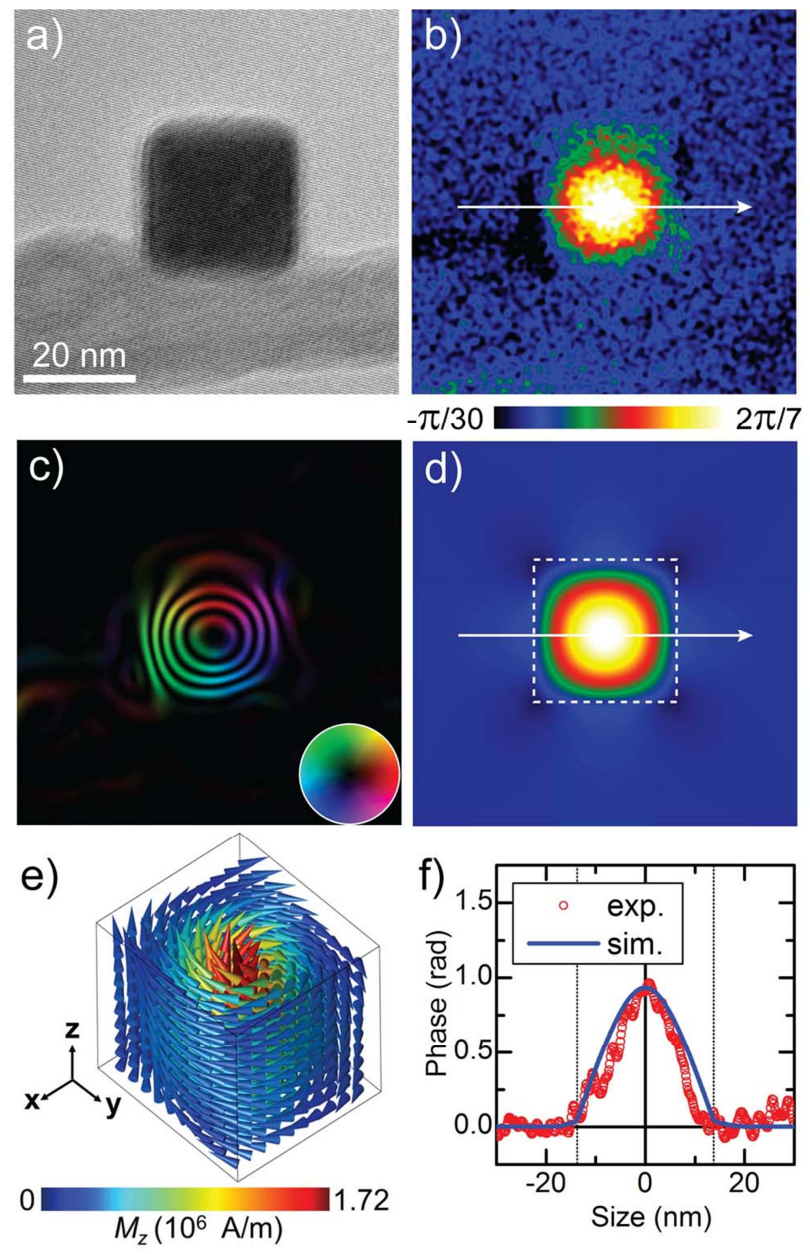

Figure 2. Magnetic electron holography vs. micromagnetic simulations of an isolated iron nanocube of $27 \times 26 \times(27 \pm 5) \mathrm{nm}^{3}$. (a) Experimental hologram and (b) resulting magnetic phase shift map. (c) Cosine of the experimental phase image corresponding to the magnetic induction flux lines (see Supporting Information). The inset color wheel indicates the direction of the magnetic induction. (d) Simulated magnetic phase shift map calculated from micromagnetic simulations (dashed box indicates the cube position). (e) 3D view of the magnetization obtained at the equilibrium state for a simulated cube of $29.5^{3} \mathrm{~nm}^{3}\left(27.5^{3}\right.$ $\mathrm{nm}^{3} \mathrm{Fe}$ core and a $1 \mathrm{~nm} \mathrm{Fe}_{3} \mathrm{O}_{4}$ shell). (f) Comparison of experimental and simulated profiles along the arrows indicated in (b) and (d) (the vertical dashed lines indicate the cube 
position).

Focusing on the uniform spin arrangement expected below the SD/V limit, a NC of $24 \times 26 \times(25 \pm 4) \mathrm{nm}^{3}$ was investigated by EH as shown in Figure 3. The resulting magnetic phase shift map with superimposed isophase contours is reported in Figure 3(b) to evidence the magnetic flux lines. While these lines are fairly aligned within the cube, they tend to curl outside in order to close the induction flux. At first sight, such features could be characteristic of a SD or a V state along the in plane [010] direction. Micromagnetic simulations are then mandatory to unambiguously distinguish between both configurations. Starting from a randomly spin orientation in a cube of similar dimension, a SD state is obtained (Fig. 3(e)). A [010] vortex state is artificially stabilized numerically using a slightly reduced exchange constant for comparison. The two profiles are then compared with the experimental one (Fig. 3(f)). The drastic difference of amplitude clearly evidences that SD is experimentally observed. A closer look at the vicinity of the edges reveals that the magnetization flares out, both on simulated and experimental mappings (Figure 3(b-d)). Such an effect is due to the inhomogeneity of the stray field and makes this SD state usually referred as flower state.Erreur ! Signet non défini.Erreur ! Signet non défini.'Erreur ! Signet non défini.'Erreur ! Signet non défini. Another example of the experimental evidence of a SD-flower state is also given in Supporting Information Figure S3 for a $22 \times 26 \times(24 \pm 4) \mathrm{nm}^{3}$ cube. While a SD state has been previously reported within an isolated faceted $50 \mathrm{~nm}$ diameter magnetite crystal,Erreur ! Signet non défini. it is the first time that a SD-flower state is quantitatively and unambiguously evidenced in a single nanomagnet as small as $25 \mathrm{~nm}$. 

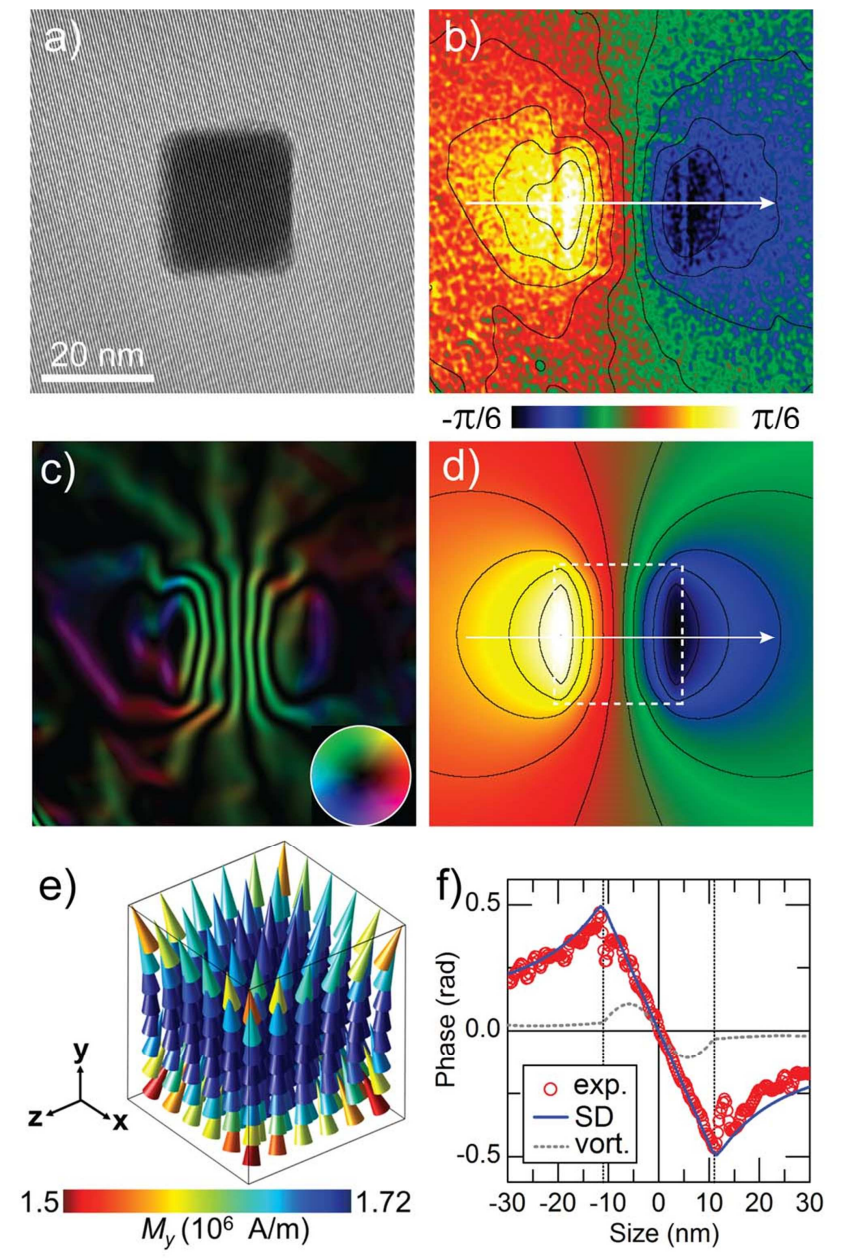

Figure 3. (a) Electron hologram of a $24 \times 26 \times(25 \pm 4) \mathrm{nm}^{3}$ Fe nanocube and (b) corresponding magnetic phase shift map with 78 mrad isophase contours (as for (e)). (c) Cosines of the experimental phase corresponding to the magnetic induction flux lines. The inset color wheel indicates the direction of the magnetic induction. (d) Magnetic phase shift map calculated from micromagnetic simulations (dashed box indicates the cube position). (e) $3 \mathrm{D}$ view of the calculated magnetization obtained at the equilibrium state for a simulated cube of a $24 \times 26 \times 24 \mathrm{~nm}^{3}$ Fe nanocube $\left(22 \times 24 \times 22 \mathrm{~nm}^{3}\right.$ Fe core surrounded with a $1 \mathrm{~nm}$ $\mathrm{Fe}_{3} \mathrm{O}_{4}$ shell). (f) Comparison of experimental and simulated profiles along the arrows indicated in (b) and (d) (the vertical dashed lines indicate the cube position). For 
comparison, the dashed line represents the profile obtained for a vortex configuration whose the core is aligned along the [010] (i.e. y) direction of the NC.

Figure 4(a) shows a hologram recorded on an intermediate $\mathrm{NC}$ of $25 \times 27 \times(26 \pm 4) \mathrm{nm}^{3}$. The extracted magnetic phase image presents a complex pattern which cannot be directly interpreted (Figure 4(b)). The micromagnetic simulation of a cube of similar dimension obtained after relaxation of a randomly spin orientation leads to a complex spin arrangement (Fig. 4(c)): its projection along the cube diagonal reveals that spins curl around $\langle 111\rangle$ direction (Fig. 4(d)). This complex configuration is therefore a vortex, the core axis of which is aligned along the diagonal of the cubes, referred hereafter as vortex $\langle 111\rangle(\langle 111\rangle$ vortex $)$. The calculated magnetic phase shift map extracted from this simulated spin arrangement is reported in Fig. 4(e) and presents the same general pattern as its experimental counterpart. On Fig. 4(f), a quantitative comparison of linear profiles evidences a good agreement between experimental and simulated profiles and evidencing the sensitivity of the EH-TEM setup, even for such a weak phase shift (less than $\pi / 5$ in the NC). A second example of a $<111>$ vortex is given in Supporting Fig. S4.

These experimental configurations and the size at which they are observed have to be compared with theoretical results. Considering nanocubes with uniaxial anisotropy, numerical studies have shown that the single-domain limit separates SD and $<001>$ vortex states without the presence of an intermediate state.Erreur! Signet non défini.Erreur! Signet non défini.Erreur ! Signet non défini. Calculations on Fe nanospheres with a cubic anisotropy proposed a hard-axis-oriented vortex as an intermediate state for diameters between 25 and 40 nm.Erreur ! Signet non défini. The present data on single-crystalline Fe nanocubes of cubic 
anisotropy demonstrate that the three $\mathrm{SD},<111>$ and $<001>$ vortices are stable configurations. In order to address more precisely the stability of each configuration, we performed additional micromagnetic simulations. We restricted our study to Fe NCs exhibiting ferromagnetic behaviour at room temperature, i.e. for mean length above $15 \mathrm{~nm}$, but with a size lower than the domain wall width (64nm for Fe).

In a first approach, simulations were carried out on NCs for which the initial spin configuration, uniform SD or $\mathrm{V}<001>$, was imposed. The resulting energies were calculated for different NC sizes and reported in Figure 5 as blue and red lines for the SD and V<001> respectively. Magnetic transition between $\mathrm{SD}$ and $\mathrm{V}<001>$ should then occur at $27 \mathrm{~nm}$, i.e. around 11 times the Fe exchange length $\left(l_{e x}{ }^{\mathrm{Fe}}=2.4 \mathrm{~nm}\right)$. SD minimizes the exchange energy for small sizes, at the expense of magneto-static energy which scales with the volume. In contrast, $\mathrm{V}<001>$ minimizes the magneto-static energy but leads to an increase of the exchange energy due to the spin disorientations between "vertical" spins in the core and "horizontal" spins at the cube periphery. When the cube size is reduced, angle between neighboring spins from the center to the periphery become larger and increase the exchange cost. 

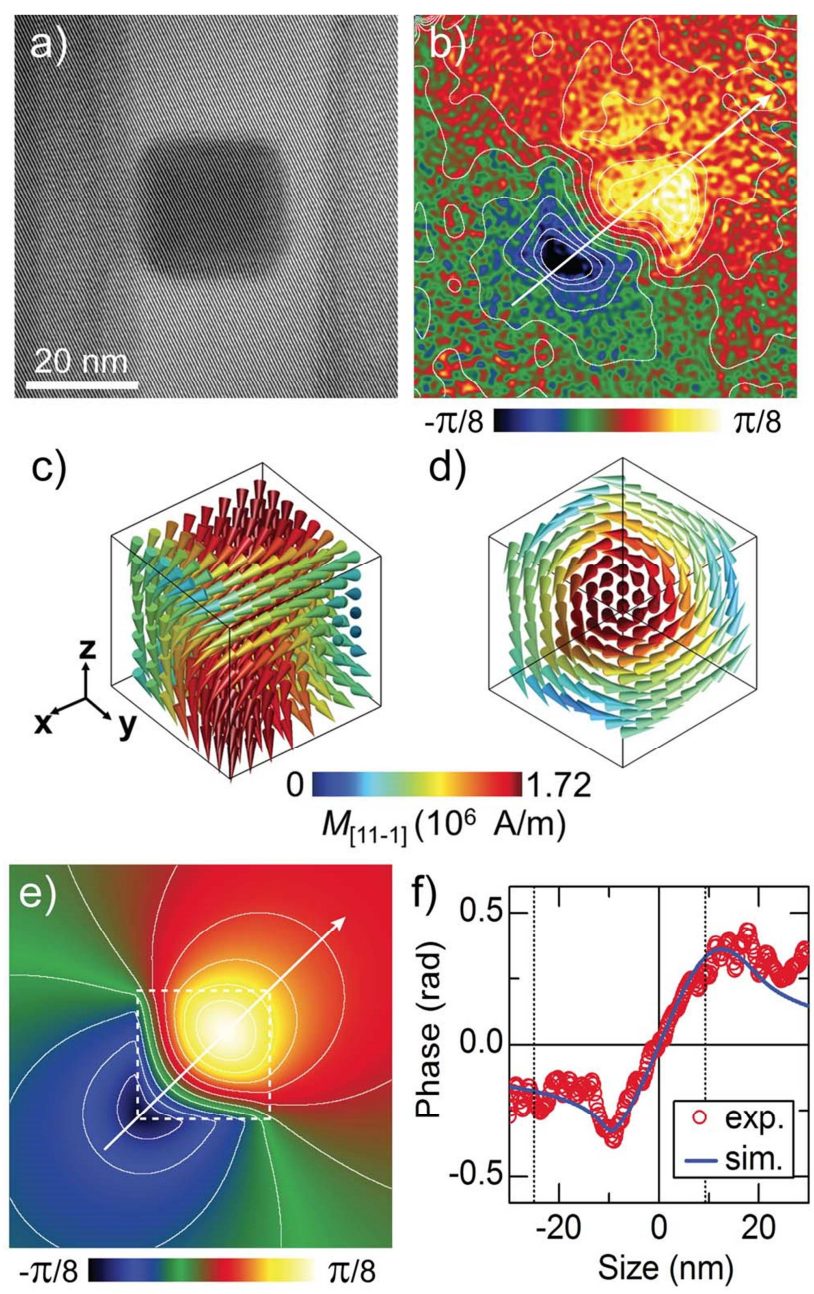

Figure 4. (a) Hologram and (b) corresponding magnetic phase shift map of a $25 \times 27 \times(26 \pm 4)$ $\mathrm{nm}^{3} \mathrm{Fe}$ nanocube with 62 mrad isophase contours. (c) 3D view of the simulated magnetization in a perfect $\mathrm{Fe}$ nanocube of $26^{3} \mathrm{~nm}^{3}\left(24^{3} \mathrm{~nm}^{3}\right.$ Fe core with a $1 \mathrm{~nm} \mathrm{Fe} \mathrm{O}_{4}$ shell) starting from an initial random distribution of moments. (d) Projection of (c) along the [11-1] direction of the cube illustrating a vortex configuration with a core-axis parallel to the $\langle 111\rangle$ direction, i.e. one of the main diagonal of the cube. (e) Calculated magnetic phase shift map with same isophase contours as (b) obtained from the simulation of a $26^{3} \mathrm{~nm}^{3}$ cube (dashed box indicates the cube position). (f) Comparison of experimental and simulated profiles along the arrows indicated in (b) and (c) (the vertical dashed lines indicate the cube position). 
This first fairly simple simulation approach was corroborated by a second method into which a random spin configuration is originally introduced. Relaxation of the spins leads to the stable configuration and the corresponding energy is plotted as dots in Figure 5. Below $25 \mathrm{~nm}$ (above $29.5 \mathrm{~nm})$, stable SD $(\mathrm{V}<001>)$ configurations are observed. This is consistent with previous measurements of $\mathrm{V}<001>$ states in $30 \mathrm{~nm}$ Fe nanocubes. ${ }^{18}$ As expected, energies are identical for vortices with [100], [010] and [001] core axis. For intermediate sizes, between 25.5 and $29 \mathrm{~nm}$, simulations evidence <111> vortex as the most stable configuration, the four equivalent directions [111], [1 $\overline{1} 1],[11 \overline{1}]$ and $[\overline{1} 11]$ leading to similar energies. In this size range where they are observed experimentally, $\mathrm{V}<111>$ have a lower energy than SD and $\mathrm{V}<001>$ configuration. This can be understood with the following argument: the core axis aligned along a <111> direction permits to accommodate along a $\mathrm{NC}$ diagonal the spin disorientation between the vortex core and its periphery. Thus the $\mathrm{V}<111>$ allows balancing exchange and magneto-static interactions at the expense of the magnetocrystalline energy which remains quite low in Fe.

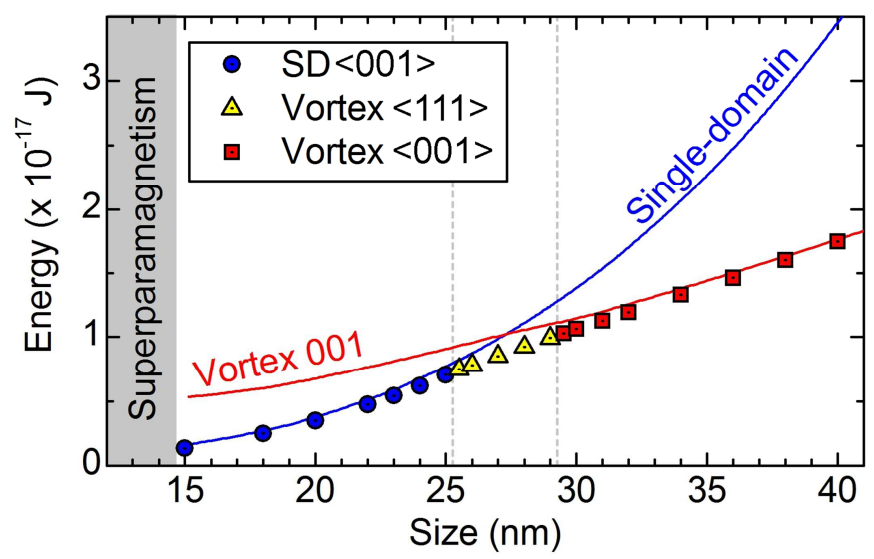

Figure 5. Calculated magnetic energy of ferromagnetic iron nanocubes (including a $1 \mathrm{~nm}$ $\mathrm{Fe}_{3} \mathrm{O}_{4}$ shell) as a function of the total cube size (range 15-40 nm). The energies were calculated once the SD or V configurations were reached. Comparison between random 
(dots) and frozen SD (blue line) or Vortex <001> (red line) initial states is reported.

Experimentally we evidenced SD, <111> and <001> vortex states for cubes of roughly 25,26 and $27 \mathrm{~nm}$ size respectively, confirming the sharp but sequential transition between these three configurations when increasing the NC size. The size range at which we observed each state was however not as large as suggested by the phase diagram of Fig.5. Particularly, the V<001> vortex of Figure 2 is not expected for the measured NC size of $27 \times 26 \times(27 \pm 5) \mathrm{nm}^{3}$, therefore we compare it with the smallest calculated NC for which $\mathrm{V}<001>$ is the ground state $\left(29.5 \mathrm{~nm}^{3}\right)$. This discrepancy might have several origins. First, the real nanocube thickness remains unknown and only a combined use of high-resolution tomography and HRTEM on a same NC could allow refining the NC dimension and could be carried out in our equipment. Secondly, the magnetic parameters used for simulation are those deduced from macroscopic measurements on $\mathrm{NC}$ assemblies and that correspond to the bulk ones. These values are however mean values of a given distributions and therefore the magnetic parameters may slightly differ from one cube to another. However the major conclusion is that, even if slight deviations from bulk prediction may occur, the quantitative comparisons between simulated and experimental profiles allow determining unambiguously the presence of a given magnetic configuration. Thus, these results demonstrate that the single-domain limit separating SD and V states is effectively in the range predicted by simulations using bulk magnetic parameters and, in addition, involves the appearance of an intermediate spin arrangement.

Here we report the spin configuration phase diagram in size-controlled single iron nanomagnets combining state of the art of magnetic electron holography experiments and micromagnetic simulations. High sensitivity imaging explicitly reveals how three different spin 
arrangements can be stabilized within a $3 \mathrm{~nm}$ window, evidencing the key importance of nanometric size control of magnetic nanoparticles. Moreover, it gives a deeper understanding of the single domain limit, which is more complex than expected with the appearance of a previously unreported $<111\rangle$ vortex state. Such a measurement opens the door to fine magnetic control of nano-objects which will find applications in fields as wild as spintronics devices, information storage or hyperthermia.

Supporting Information

Details of the chemical synthesis of $\mathrm{Fe}$ nanocubes, structural characterization, electron holography experiments and analysis, 3D micromagnetic simulations, histograms of nanocube size and squareness ratio, additional experimental evidences of SD flower, $\langle 001\rangle$ and $\langle 111\rangle$ vortices. This material is available free of charge via the Internet at http://pubs.acs.org.

\section{Corresponding Author}

* Blon, T.: thomas.blon@insa-toulouse.fr

* Gatel, C.: gatel@cemes.fr

Notes

The authors declare no competing financial interest. 
Acknowledgements

The authors acknowledge M. Respaud for fruitful discussion and careful reading of the manuscript. C.G., E.S., B.W. acknowledge the French National Research Agency under the "Investissement d'Avenir" program reference No. ANR-10-EQPX-38-01" the "Conseil Regional Midi-Pyrénées", the European FEDER for financial support within the CPER program and the European Union under the Seventh Framework Program under a contract for an Integrated Infrastructure Initiative Reference 312483-ESTEEM2. This work was supported by the French national project EMMA (ANR12 BS10 013 01) and the French microscopy network METSA.

\section{References}

${ }^{1}$ Parking, S.S.P.; Hayashi, M.; Thomas, L. Science 2008, 320, 190-194

${ }^{2}$ Biziere, N.; Gatel, C.; Lassalle-Balier, R.; Clochard, M.-C.; Wegrowe, J.-E.; Snoeck, E. Nano Lett. 2013, 13, 2053-2057

${ }^{3}$ Terris, B.D.; Thomson, T. J. Phys. D: Appl. Phys.2005, 38, R199-R222

${ }^{4}$ Liakakos, N.; Blon, T.; Achkar, C.; Vilar, V.; Cormary, B.; Tan, R.P.; Benamara, O.; Chaboussant, G.; Ott, F.; Warot-Fonrose, B.; Snoeck, E.; Chaudret, B.; Soulantica, K.; Respaud, M. Nano Lett. 2014, 14, 3481-3486

${ }^{5}$ Poudyal, N.; Ping Liu, J. J. Phys. D: Appl. Phys. 2013, 46, 043001 1-23

${ }^{6}$ Gandha, K., Elkins, K., Poudyal, N., Liu, X., Ping Liu, J. Sci. Rep., 2014, 4, 5345 1-5

${ }^{7}$ Ho, D.; Sun, X.; Sun, S. Acc. Chem. Res. 2011, 44, 875-882 
${ }^{8}$ Yang, Y.; Liu, X.-L.; Yi, J.-B.; Yang, Y.; Fan, H.-M.; Ding, J. J. Appl. Phys. 2012, 111, $0443031-9$

${ }^{9}$ Pribiag, V.S.; Krivorotov, I.N.; Fuchs, G.D.; Braganca, P.M.; Ozatay, O.; Sankey, J.C.; Ralph, D.C.; Buhrman, R.A. Nat. Phys. 2007, 3, 498-503

${ }^{10}$ Schabes, M.E. ; Bertram, H.N. J. Appl. Phys. 1988, 64, 1347-1357

${ }^{11}$ Rave, W.; Fabian, K.; Hubert, A. J. Magn. Magn. Mater. 1998, 190, 332-348

${ }^{12}$ Hertel, R.; Kronmüller, H. J. Magn. Magn. Mater. 2002, 238, 185-199

${ }^{13}$ Muxworthy, A.R.; Williams, W.; Roberts, A.P.; Winklhofer, M.; Chang, L.; Pósfai, M. Geochem., Geophys., Geosyst. 2013, 14, 5430-5441

${ }^{14}$ Betto, D.; Coey, J.M.D. J. Appl. Phys. 2014, 115, 17D138 1-3

${ }^{15}$ Kakay, A.; Varga, L.K. J. Appl. Phys. 2005, 97, 083901 1-4

${ }^{16}$ Snoeck, E.; Dunin-Borkowski, R.E.; Dumestre, F.; Renaud, P. ; Amiens, C.; Chaudret, B.; Zurcher, P. Appl. Phys. Lett. 2003, 82, 88-90

${ }^{17}$ Akhtari-Zavareh, A.; Carignan, L.P.; Yelon, A.; Ménard, D.; Kasama, T.; Herring, R. ; DuninBorkowski, R.E.; McCartney, M.R.; Kavanagh, K.L. J. App. Phys. 2014, 116, 023902 1-11

${ }^{18}$ Snoeck, E.; Gatel, C.;; Blon, T.;; Carrey, J.; Respaud, M.; Chaudret, B. Nano Lett. 2008, 8, $4293-4298$

19 Lacroix, L.-M.; Lachaize, S.; Hue, F.; Gatel, C.; Blon T.; Tan, R.P.; Carrey, J.; WarotFonrose B.; Chaudret B. Nano Lett. 2012, 12, 3245-3250 
${ }^{20}$ Hÿtch, M.J.; Dunin-Borkowski, R.E.; Scheinfein, M.R.; Moulin, J.; Duhamel, C.; Mazaleyrat, F.; Champion, Y. Phys. Rev. Lett. 2003, 91, 257207 1-4

${ }^{21}$ He, K.; Ma, F.-X.; Xu, C.-Y.; Cumings, J. J. App. Phys. 2013, 113, 17 B528 1-3

${ }^{22}$ Varón, M.; Beleggia, M.; Kasama, T.; Harrison, R.J.; Dunin-Borkowski, R.E.; Puntes, V.F.; Frandsen, C. Sci. Rep. 2014, 3, 1234 1-5

${ }^{23}$ Thomas, J.M.; Simpson, E.T.; Kasama, T.; Dunin-Borkowski, R.E. Acc. Chem Res. 2008, 41, 665-674

${ }^{24}$ Beleggia, M.; Lau, J.W.; Schofield, M.A.; Zhu, Y.; Tandon, S.; De Graef, M. J. Magn. Magn. Mater. 2006, 301, 131-146

${ }^{25}$ Singh, D.K.; Krotkov, R.; Tuominen, M.T. Phys. Rev. B 2009, 79, 184409 1-9

${ }^{26}$ Shinjo, T.; Okuno, T.; Hassdorf, R.; Shigeto, K.; Ono, T. Science 2000, 289, 930-932

${ }^{27}$ Amos, N.; Ikkawi, R.; Haddon, R.; Litvinov, D.; Khizroev, S. Appl. Phys. Lett. 2008, 93, 203116 1-3

${ }^{28}$ Fraile Rodríguez, A.; Kleibert, A.; Bansmann, J.; Voitkans, A.; Heyderman, L.-J.; Nolting, F. Phys. Rev. Lett. 2010, 104, 127201 1-4

${ }^{29}$ Wachowiak, A.; Wiebe, J.; Bode, M.; Pietzsch, O.; Morgensten, M.; Wiesendanger, R. Science 2002, 298, 577-580

${ }^{30}$ Lacroix, L.-M. ; Lachaize, S.; Falqui, A.; Respaud, M.; Chaudret, B. J. Am. Chem. Soc. 2009, $131,549-557$ 
${ }^{31}$ Donahue, M.J.; Porter, D.G. OOMMF User's Guide, Version 1.0, Interagency Report NISTIR 6376, National Institute of Standards and Technology, Gaithersburg, MD (1999) 